\title{
Cyber-Physical Museum Exhibits Based on Additive Technologies, Tangible Interfaces and Scientific Visualization
}

\author{
K.V. Ryabinin'1,A, M.A. Kolesnik2,B, A.I. Akhtamzyan3,, , E.V. Sudarikova4,C \\ A Perm State University \\ B Perm Regional Museum \\ c State Darwin Museum \\ ${ }^{1}$ ORCID: 0ooo-0002-8353-7641, kostya.ryabinin@gmail.com \\ 2 ORCID: 000o-0002-4424-4723, kolesnik.ma@outlook.com \\ 3 ORCID: 0000-0002-8367-9156, darnhalm@gmail.com \\ 4 ORCID: 0000-0001-8384-9832, elsud@darwinmuseum.ru
}

\begin{abstract}
This paper is devoted to the new methods of organizing interactive museum exhibits by means of additive technologies, programmable microelectronics and multimedia. Traditionally museum exhibition is distanced from the visitor appearing just as a visual image. But new trend is to let the visitor inside the exhibition, providing different forms of interactions. Modern museums constantly search for the new ways of presenting cultural and natural heritage taking into account that exhibitions should be at the same time scientifically accurate, attractive, memorable and, ideally, accessible for the wide audience including disabled people. In this regard, tangible user interfaces based on the Internet of Things and combined with the scientific visualization build up very promising set of technologies that brings to live cyberphysical museum exhibits. These exhibits are an alloy of physical museum items with virtual multimedia content, providing the visitor a unique experience of interactivity. The key feature of cyber-physical exhibits is their tangibility, whereby they become accessible for visually impaired people and deliver much more information for the regular visitors.

There are a lot of successful attempts to build cyber-physical exhibits within the museum space. However, there is a lack of methodological basis for this, as well as the lack of highlevel tools to seamlessly integrate related technologies into the existing museum infrastructure. In this paper we propose using ontology-driven adaptive multiplatform scientific visualization system SciVi as a software basis for cyber-physical museum exhibits. This system was previously successfully used in solving problems related to steering hardware and software solvers in different application domains, monitoring lightweight robotics systems and supporting custom hardware human-machine interfaces. So, it contains necessary mechanisms to adapt to the third-party digital infrastructure (including the museum one) and build all the required middleware and visualizers within it.

We tested our approach by developing two cyber physical exhibits: tangible bonobo skull in the State Darwin Museum (Moscow) and tangible titanophone skulls in the Museum of Permian Antiquities (Perm). Bonobo exhibit appears to be a custom joystick in a form of corresponding skull to steer the Sketchfab-rendered 3D model of bonobo monkey head. Titanophone exhibit allows visitors to discover the age variability of titanophone synapsid in an interactive way.
\end{abstract}

Keywords: museum, scientific visualization, Internet of Things, tangible interface, cyber-physical system, additive manufacturing, ontology engineering. 


\section{Introduction}

The idea of tangible user interfaces (TUI) was first introduced by Hiroshi Ishii in 1997 [1]. Nowadays, with the rise of the Internet of Things (IoT) technologies [2], TUI experience the rebirth on the basis of modern programmable microelectronics. Alloy of TUI and IoT opens a gate for so-called cyber-physical systems (CPS) [3] systems, where virtual and real worlds are tightly interconnected. Real and virtual objects in these systems complement each other: manipulations over real objects affect virtual ones and vice versa. CPS is all about dissolving the border between the real world and cyberspace as much, as it is possible with the software and hardware means used. Ideally, this border should be imperceptible for the user. CPS enable socalled Fourth Industrial Revolution [4] opening for the mankind new ways of human-machine interaction, and as the result, new horizons of digital technologies.

One of the many practical uses of CPS are interactive exhibits in museums. Currently, the set of IoT-based technologies related to museums is combined into socalled Smart Museum concept [5]. Smart Museum covers methods and means to monitor the visitors' activity, implement the indoor navigation and compose the interactive exhibitions. In this sense, building CPS with museum items and related digital content is a logical step in Smart Museum development.

Organizing the museum space in the form of CPS can significantly increase the attractiveness and memorability of the exposition, as well as provide visitors with a wide variety of information and, as a result, expand the museum's potential as a scientific, cultural and educational platform. However, the practical implementation of CPS within existing museums faces notable difficulties:

1. Seamless integration with the existing digital infrastructure of the museum: new digital means (like IoT-based TUI or new multimedia content) should reuse the software and hardware already installed in the museum. The digital infrastructure of the museum should be enriched by new technologies, not replaced in their favor.

2. Lack of high-level tools to deploy and control CPS taking into account small number of IT specialists in the museum staff.

In our previous work we suggested methods and high-level means to automate hardware human-machine interfaces development based on ontology engineering and IoT technologies [6], as well as used the IoT technologies and scientific visualization to create interactive museum exhibits [7].

The aim of the current work is to synthesize the results of previous studies and formulate on their basis the general concept of creating museum CPS. The proposed concept is applied in two practical cases by creating cyber-physical exhibits in the State Darwin Museum (Moscow, Russia) and Museum of Permian Antiquities (Perm, Russia).

\section{Cyber-Physical Museum Exhibits Concept}

Normally the museum items may not be touched. However, museums are constantly searching for the ways to dissolve the showcase glass and to allow the visitor to contact with the exhibits without jeopardizing the physical condition of cultural and / or natural heritage. The closer this contact will be, the more information the visitor will obtain.

Classical technologies easily extend the visual contact with the exhibit by the audio channel, as well as present some digitized information in a form of so-called "live label" - a monitor (or even touchscreen) displaying related multimedia content near 
the exhibit showcase. However, these traditional means lack tangibility. In this sense, CPS technologies are the way to enrich the museum exhibition with haptics and thereby enlarge the spectrum of information received by the visitor. Moreover, graspable items support museum inclusion: tactile contact allows blind and visually impaired persons to learn much more about the exhibits than just hearing the traditional voiceovers.

As stated in [8], "An ideal museum will be one where visitors can appreciate the charm of tactile culture with hands, fingertips and whole body, not simply learn through looking at exhibits".

There are many examples of successful usage of tangible interfaces within museums, which quality in pragmatic and hedonic aspects was proven in practice [9-15]. Wide range of technologies is utilized while building cyber-physical exhibits, from programmable microcontrollers with custom developed circuits (like in [14, 15]) to complicated prefabricated touch- and display surfaces (like in $[11,12])$.

The essence of the museum CPS is the concept behind it: the idea of the cyberphysical exhibit. The modern technologies are just a toolset to embody this idea. But analyzing the ways the most notable ideas were implemented (like, for example, the results obtained by the authors of [9-15]) we can point out the lack of standards and the absence of common methodology for creating museum CPS.

Trying to fill this methodological gap, we propose technological stack to potentially automate or at least simplify the creation of cyber-physical museum exhibits. Related to the museum space, we suggest the following structure of CPS:

1. Physical objects: museum items with IoT-based sensors, which can detect interactions with the visitors, as well as actuators (motors, solenoids, etc.), which can provide tangible feedback. Hereafter this part is denoted as $P_{1}$.

2. Virtual objects: multimedia content that enriches the museum items by visualizations, voiceovers, sounds, videos, etc. Software implementation should be based on the scientific visualization techniques to ensure the required level of scientific reliability of the information provided to the visitor. Hereafter this part is denoted as $P_{2}$.

3. Display: device that shows the virtual objects. It can be a stationary monitor or projector (if required, with sound playing system on board) installed in the museum, or personal mobile device of the visitor (smartphone or tablet computer). Hereafter this part is denoted as $P_{3}$.

Practical implementation of such CPS kind faces the following problems, which solutions are proposed as part of the formulated concept.

First of all, most of the museum items, due to their fragility, disrepair and / or uniqueness cannot be equipped with any electronic or electromechanical devices. $\mathrm{Al}$ so, it is often not possible to organize direct (for example, tactile) interaction of visitors with these items. In this case we propose build CPS physical objects from highquality reconstructions of museum items. The creation of such reconstructions can be automated by additive manufacturing [16] using 3D scanners and $3 \mathrm{D}$ printers.

Secondly, nowadays museums already have some multimedia content presented by different types of displays: "live labels" (monitors displaying supplementary materials about the exhibits), multimedia stands (for example, virtual exhibits with gesture-based interface powered by optical gesture detection systems like MS Kinect), interactive kiosks (displaying interactive materials or providing mini-games related to the exhibits) and so on. Content and equipment operate inside a certain digital infrastructure deployed in the museum. The corresponding museum workers responsible for the support of this infrastructure are used to the technologies involved and are aware of the content they have. So, it is crucial to reuse these technol- 
ogies and content by introducing the CPS to reduce financial costs and to lower the entry threshold for employees. In other words, introduction of CPS should enrich the existing digital infrastructure, not replace it. This in turn requires high-level adaptive tools to organize CPS, which enable integration of different TUI with existing content management systems and visualization means (including scientific visualization software).

As a toolbox that meets the specified requirements, it is proposed to use the SciVi adaptive multiplatform scientific visualization system developed during the previous studies [6]. This system's behavior is fully governed by ontology knowledge base that ensures its adaptivity and scalability [6]. The possibility of using SciVi as the basis for transforming existing digital museum infrastructures into full-fledged CPS is due to the following system functions available to users through a high-level graphical interface:

1. Automated integration mechanisms (including bidirectional communication) with third-party data sources, including static file storages and dynamic data generators (software and hardware solvers) [17].

2. Extensible set of customizable preprocessing mechanisms for the visualized data [17].

3. Extensible set of customizable mechanisms, visual objects and graphical scenes for the data rendering [17].

4. Customizable mechanisms for communication with custom hardware humanmachine interfaces [6].

5. Automated generation of lightweight SciVi clones, which can run as firmware for electronic devices within IoT ecosystem [7].

6. Middleware operation mode, in which SciVi acts as a proxy to enable various combinations of communication of different hardware within IoT ecosystem, different solvers, generating the data and different visualizers, rendering these data [6].

The above-mentioned features allow SciVi to act as a software "glue" to integrate IoT devices, data generators and data storages, as well as data visualizers into a solid ecosystem, in particular, into CPS. In is worth noting, that in case some of the CPS elements already exist inside the museum digital infrastructure, they can be taken unchanged thanks to the adaptive mechanisms of SciVi. Only the missing elements should be created from scratch or bought as factory-made modules. This enables seamless integration of CPS into the museum exhibition.

The proposed concept has been put into practice by creating various cyber-physical museum exhibits. The two most interesting cases so far are described below.

\section{3. "Bonobo" Cyber-Physical Exhibit}

Cyber-physical exhibit "Bonobo" is a part of Bonobo photo exhibition [18] in State Darwin Museum, Moscow, Russia. This exhibit is based on the 3D model of the monkey bonobo (Pan paniscus Schwarz, 1929) skull, created by 3D scanning the original skull in Royal Museum for Central Africa, Tervuren, Belgium [19]. The rendering of this model is shown in the Fig. 1. It is accessible on the Sketchfab cloud service [20] one of the largest online platforms for publishing, sharing, discovering, buying and selling 3D graphics content. 


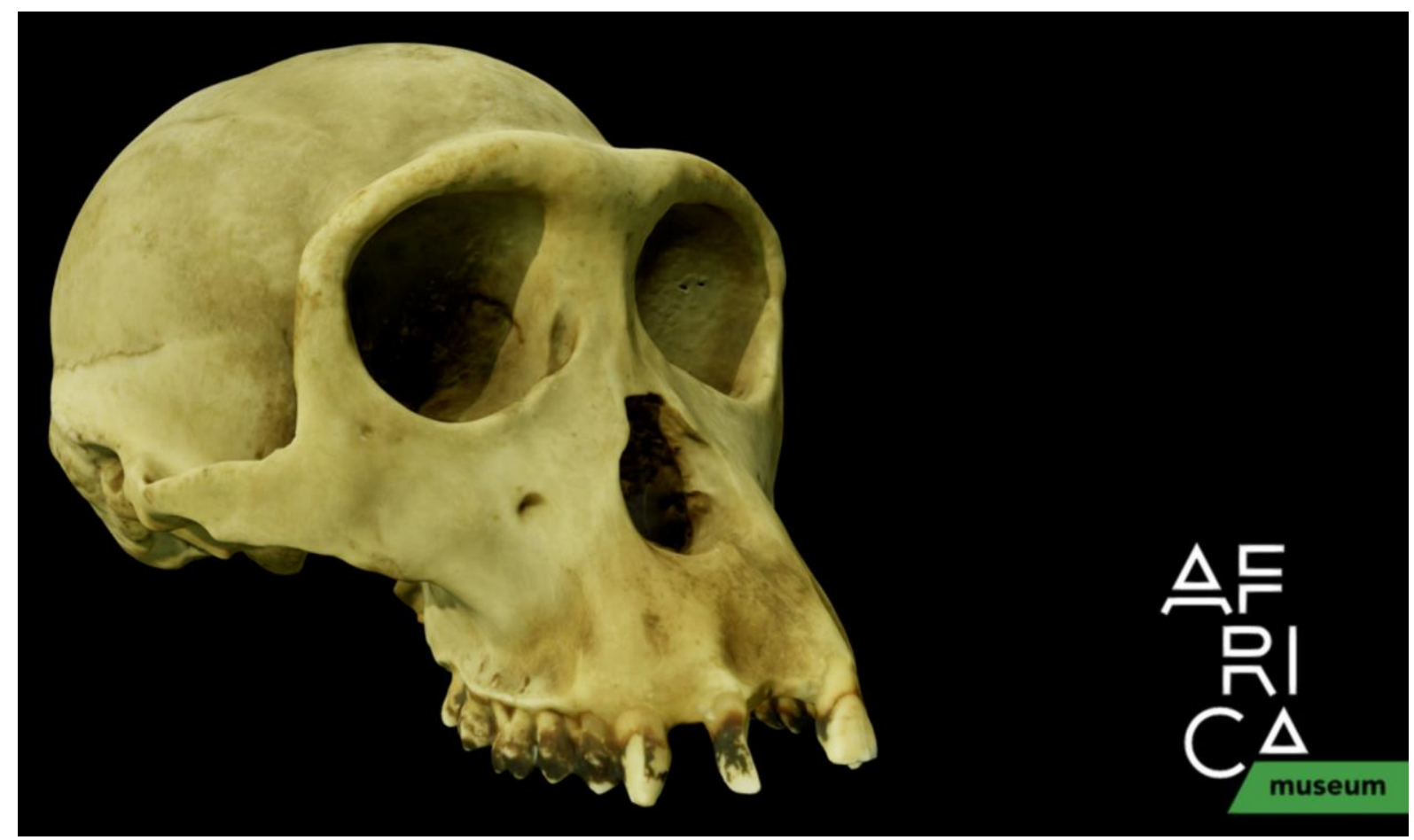

Fig. 1. Pan paniscus skull by Royal Museum for Central Africa on Sketchfab.

Sketchfab is widely used in the State Darwin Museum as a service to display $3 \mathrm{D}$ models of different exhibits. For the Bonobo photo exhibition, special visualization is composed using Sketchfab capabilities: skull model is combined with semitransparent model of the alive individual. Most interesting anatomical features on the skull are pinned and annotated with textual information and corresponding voiceovers. The process of model preparation is shown in the Fig. 2. In terms of museum CPS, which structure was described in the Section 2, this content corresponds to $P_{2}$ (virtual part) of the exhibit.

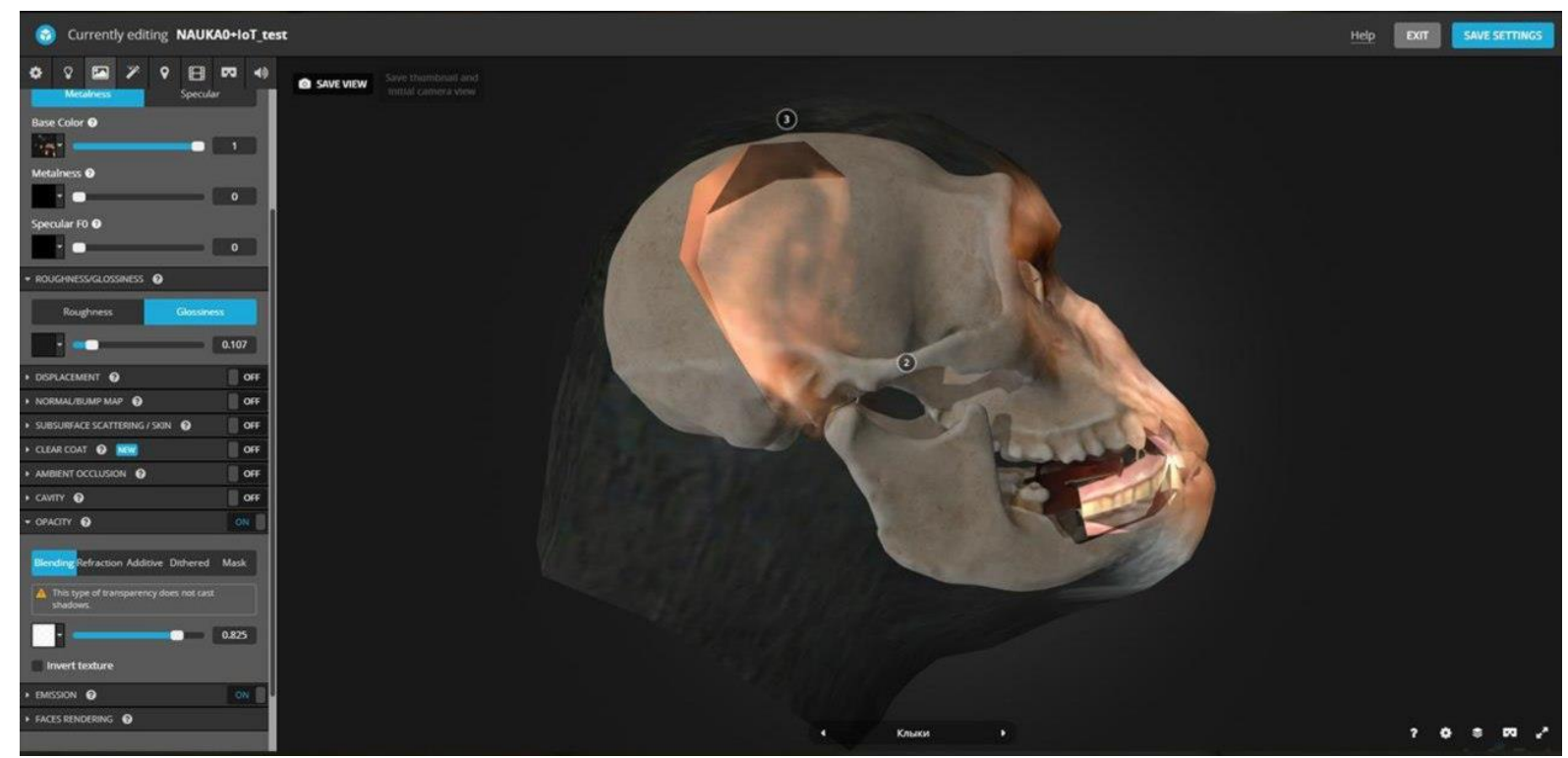

Fig. 2. Set up of key pins with annotations and the general setting of the $3 \mathrm{D}$ model on Sketchfab. 
The $P_{3}$ (display part) of CPS was already presented in State Darwin Museum: it is a kiosk with a monitor, WiFi access and WebGL-capable Web browser. This kiosk fits ideally to display models using standard Sketchfab player.

So, the only missing part of CPS was $P_{1}-$ physical object. It was created by $3 \mathrm{D}$ printing bonobo skull and using this $3 \mathrm{D}$ print as a shell for IoT device. As per the idea of this exhibit, rotation of the $3 \mathrm{D}$ printed skull should be tracked and synchronized with the rotation of the $3 \mathrm{D}$ model on the screen, while touching the anatomical key points of the skull should trigger corresponding annotations and voiceover playbacks. So, 3D printed bonobo skull should play a role of tangible custom "joystick" for the Sketchfab visualizer, allowing visitors to explore real and virtual models simultaneously.

To achieve this functionality, IoT device incorporates inertial measurement unit (IMU) GY-85 and push buttons connected to the ESP8266 WiFi-enabled microcontroller powered by $1200 \mathrm{mAh}$ accumulator. The photo of the device is shown in Fig. 3 .

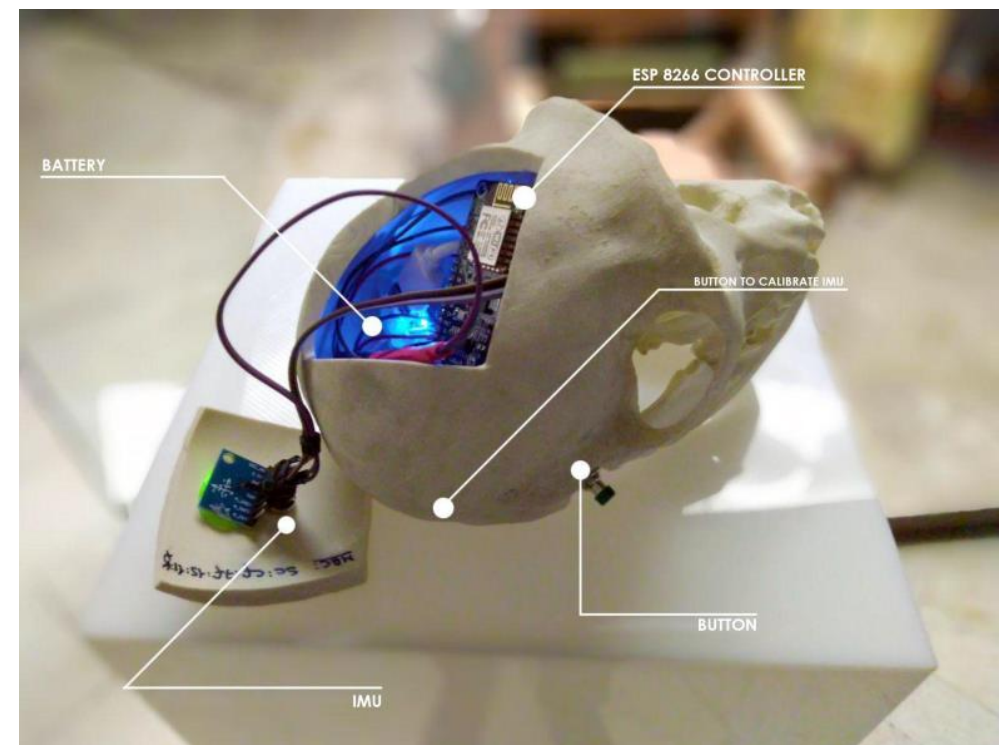

Fig. 3. IoT device the "Bonobo" cyber-physical exhibit is based on.

SciVi system was used to automate the creation of two software components: firmware for the IoT device and middleware that transmits commands from the IoT device to Sketchfab service. The operating schema is shown in the Fig. 4. 


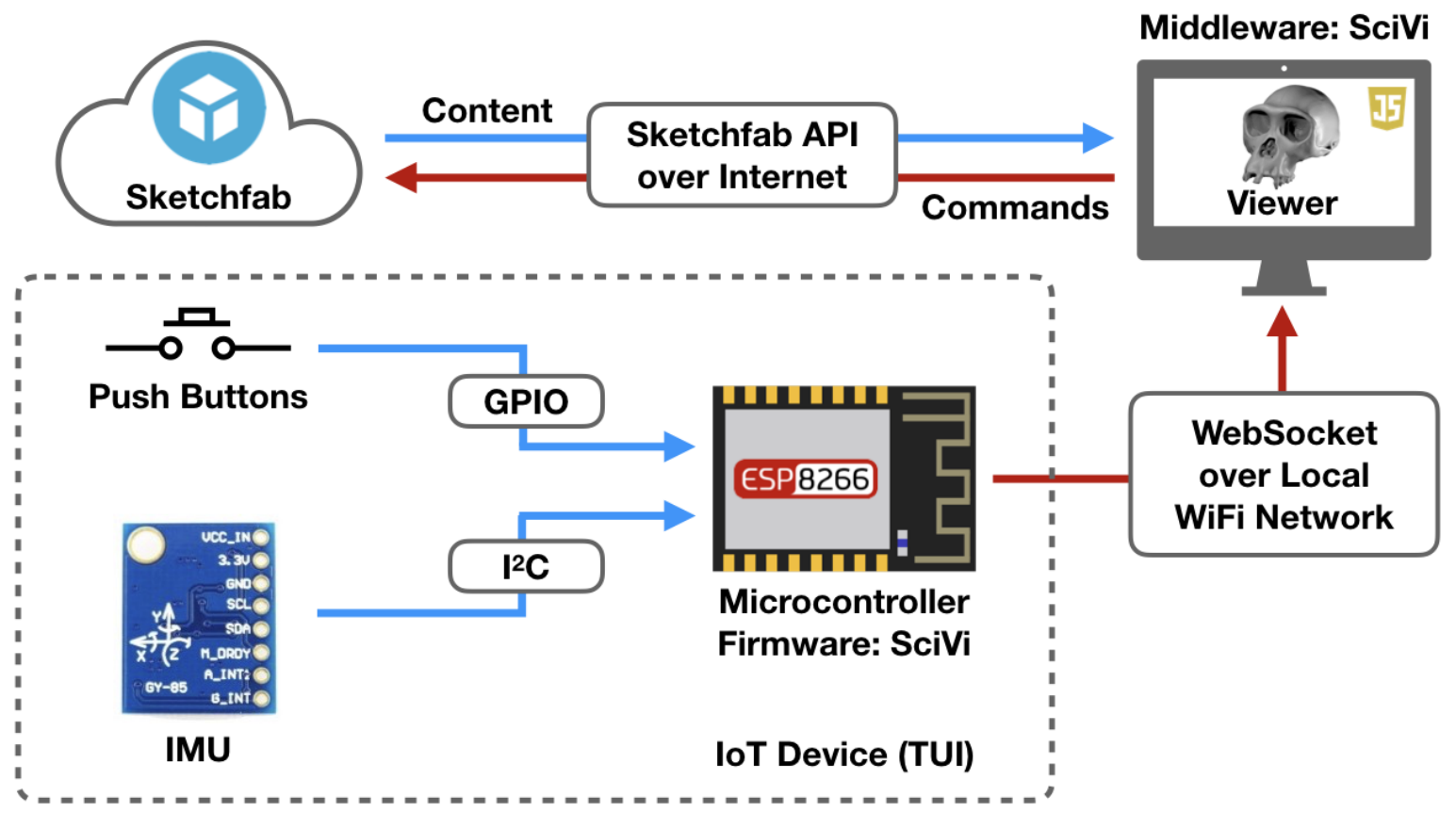

\section{$\longrightarrow$ Data Link $\longrightarrow$ Control Link}

Fig. 4. Operating schema of the "Bonobo" cyber-physical exhibit. steps:

The firmware for ESP8266 (generated by SciVi in $\mathrm{C}++$ ) has the following

1. Connect to the WiFi using SSID and password stored in the data memory (these settings are made in SciVi when tuning the firmware generation).

2. Start main loop:

a. Poll the push buttons connected via GPIO pins.

b. Obtain acceleration and angular velocity measured by the IMU.

c. Compose data from (2) into the orientation quaternion using Mahony filter [21].

d. Transmit quaternion and numbers of pushed buttons as JSON message via WebSocket connection over WiFi to the middleware.

The middleware (generated by SciVi in JavaScript, HTML5 and CSS as a standalone Web page) accomplishes the following:

1. By loading:

a. Connect to Sketchfab via Internet utilizing Sketchfab Viewer API [22], load the viewer and display the $3 \mathrm{D}$ model of bonobo head (skull and semitransparent flesh).

b. Connect to the IoT device via local WiFi utilizing standard WebSocket API of the browser. Currently, static IP address is used for the IoT device; museum router is responsible to assign it. However, client-side network discovery features may be easily added in the future if required.

2. By receiving the message via WebSocket from the IoT device:

a. If one of the buttons is pushed, activate corresponding annotation of the 3D model using Sketchfab Viewer API and play the corresponding voiceover using HTML5 audio API.

b. If orientation is changed, apply it to the $3 \mathrm{D}$ model using Sketchfab Viewer API. 
Technical details about the Sketchfab API functions utilized are described in our Sketchfab blog post [23]. To make the generation of the middleware possible within SciVi, the needed subset of Sketchfab API was described in the middleware ontology [6].

The photo of the exhibit is shown in the Fig. 5. The exhibit in action can be viewed in the video, Fig. 6.

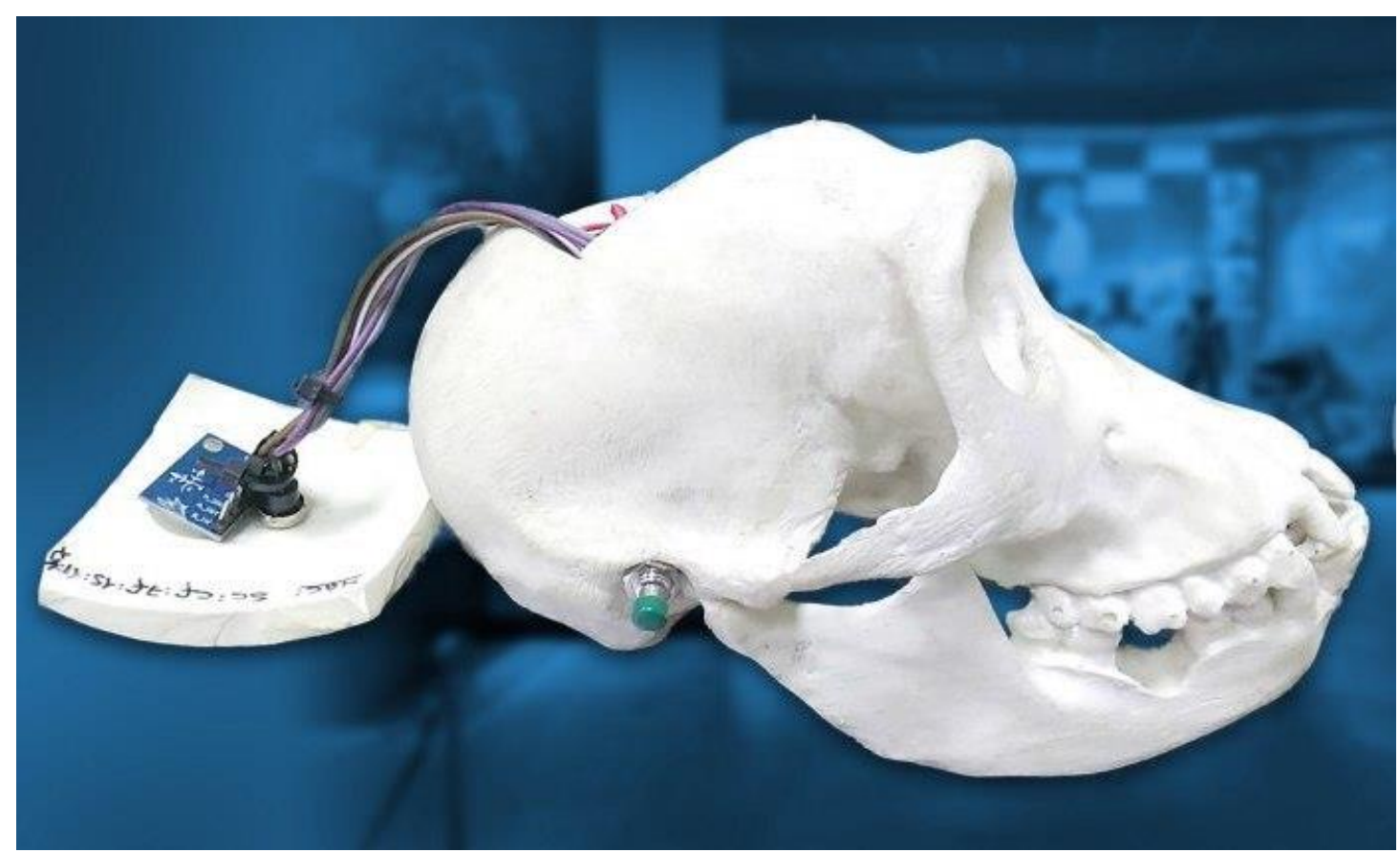

Fig. 5. Photo of the "Bonobo" cyber-physical exhibit.



Fig. 6. "Bonobo" cyber-physical exhibit in action. 


\section{4. "Titanophone" Cyber-Physical Exhibit}

The main exhibition of the Museum of Permian Antiquities contains anatomic recast of titanophone (Titanophoneus potens Efremov, 1938), large predatory synapsid of Dinocephalia order, lived in the Middle Permian. This order is characterized by significant intraspecific variation: the differences between young and adult individuals are sufficient to take them for different species, and even different genera. Efremov described titanophone in 1938 by two skeletons: No. 157/1, Paleontological Institute, Russian Academy of Sciences [24] (hereafter this skeleton is denoted as No. 157/1) and No. 157/3, Paleontological Institute, Russian Academy of Sciences (hereafter denoted as No.157/3). Titanophone has such a wide age polymorphism that Orlov even attributed No. 157/3 to a separate genus Doliosauriscus adamanteus Orlov, 1958. However, Ivakhnenko proved that No. 157/3 is in fact an adult titanophone [25].

Museum of Permian Antiquities possesses the titanophone recast created under supervision of Ivakhnenko that shows middle-age animal. But this recast does not give an idea of age-related variability. To fill this gap, we decided to create cyberphysical exhibit that will show the age differences in an interactive way using the shape morphing technique to visualize the growing process as a transition between the skulls of young and adult titanophones.

The research of titanophone paleobiology was conducted [26]. During this research, the skeletons belonging to Titanophoneus genus were measured. The skull measurements of No. 157/1 and No. 157/3 are presented in the Fig. 7.



a

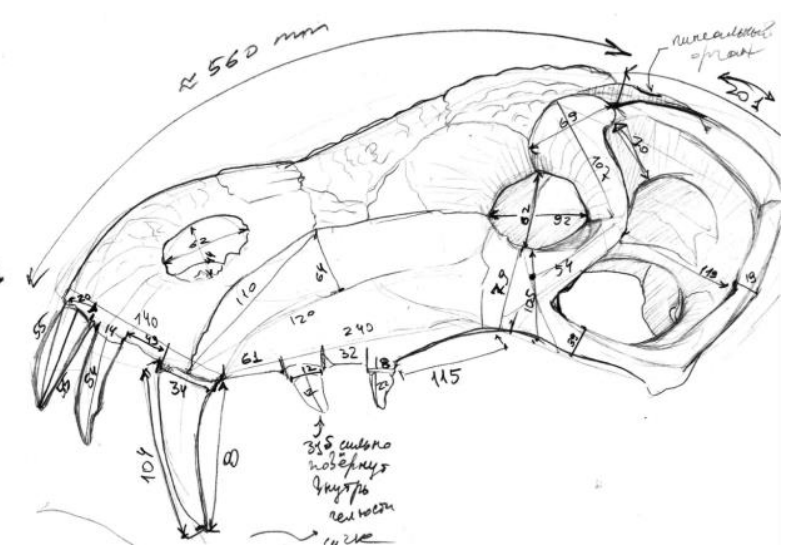

$\mathrm{b}$

Fig. 7. Skull sketches with measurements: No. 157/1 (a) and No. 157/3 (b).

Based on the sketches and measurements done, the scientifically accurate $3 \mathrm{D}$ models of No. 157/1 and No. 157/3 were created using Blender 3 D editor. The Fig. 8 demonstrates the model creation process. It must be noted, that both No. 157/1 and No. 157/3 3D models are made with the same topology (the number of vertices and connections of these vertices are the same for both models), which enables trivial morphing based on the interpolation of vertex positions. This is why we created the models manually and didn't use 3D scanning in this task. 


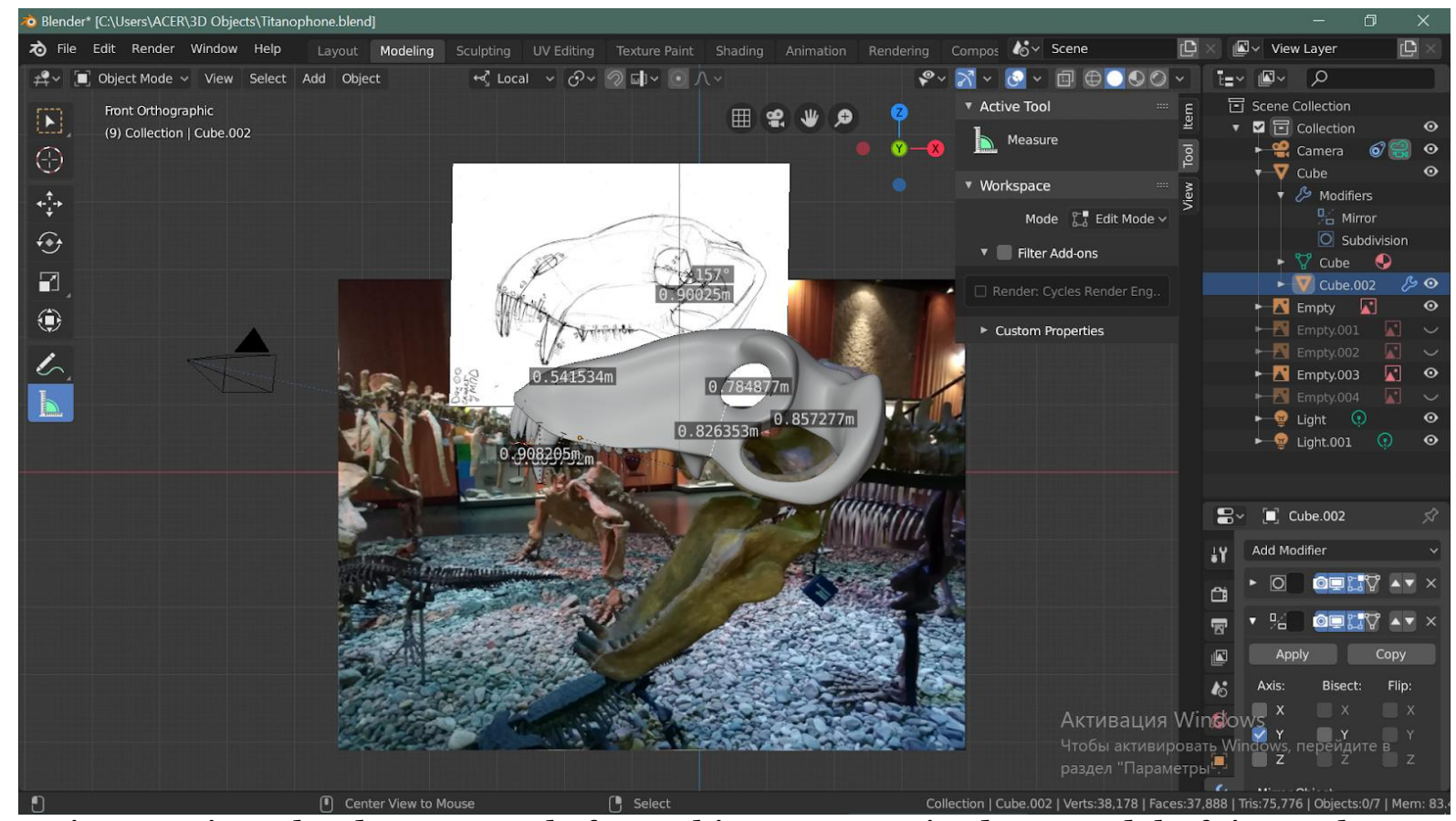

Fig. 8. Using Blender v2.8 tools for making correct-sized 3D model of titanophone skulls.

The $P_{1}$ (physical part) of the exhibit consists of the two created models of titanophone skulls printed by the modified Anet A8 3D printer. They are placed on the platforms at a distance of $40 \mathrm{~cm}$ from each other. The platform with No. 157/1 print is equipped with the IoT device, that constantly measures distance to the nearest obstacle. This distance is then interpreted as "age" of titanophone, that should be displayed to the visitor in a form of $3 \mathrm{D}$ model $\left(P_{2}\right.$, virtual part of the exhibit), rendered as a morphing from No. $157 / 1$ to No. $157 / 3$. The visitor can place a hand between the skulls altering the distance measured and exploring in such a way the ontogenetic changes of the animal. As the skulls presented are in fact reproducible prints, they can be touched. So, the visitor can not only discover the ontogenesis of titanophone, but also haptically examine the skulls this animal was described by.

The IoT device consists of distance measurement sensor and ESP8266 microcontroller powered by $1200 \mathrm{mAh}$ accumulator or optionally by $5 \mathrm{~V}$ power line. We tested two range sensors: time-of-light VL53LoX and ultrasonic HC-SRo4. While ultrasonic sensor is 4 times cheaper, both work pretty well. However, the time-of-light sensor ensures better accuracy and is more stable in measurements. Also, it possesses narrower radiation pattern that reduces false measurements when the visitor is close to the exhibit: ultrasonic sensor sometimes detects other parts of visitors' body, not just hand between the skulls, which leads to incorrect results and lower quality of experience. Time-of-light sensor has no such problem and therefore performs better. The photo of the device is shown in Fig. 9. 


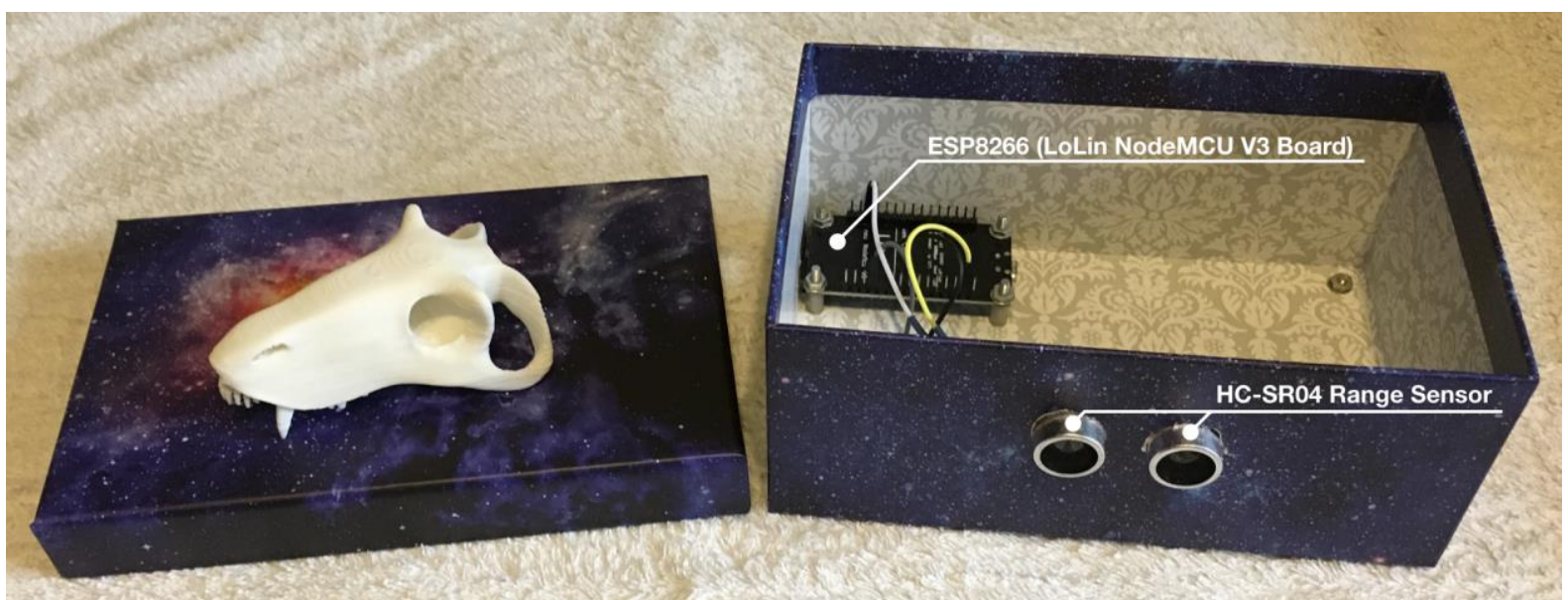

Fig. 9. The physical part of "Titanophone" cyber-physical exhibit: 3D-printed skull of young titanophone individual on the platform's roof (left) and electronic components inside the platform (right).

The $P_{3}$ (display part) of the exhibit is normally a lightweight computer with a monitor installed in the museum (for example, Raspberry Pi can be used). But under certain circumstances, it may be even the visitor's personal mobile device.

The operating schema of the entire cyber-physical exhibit is shown in the Fig. 10.

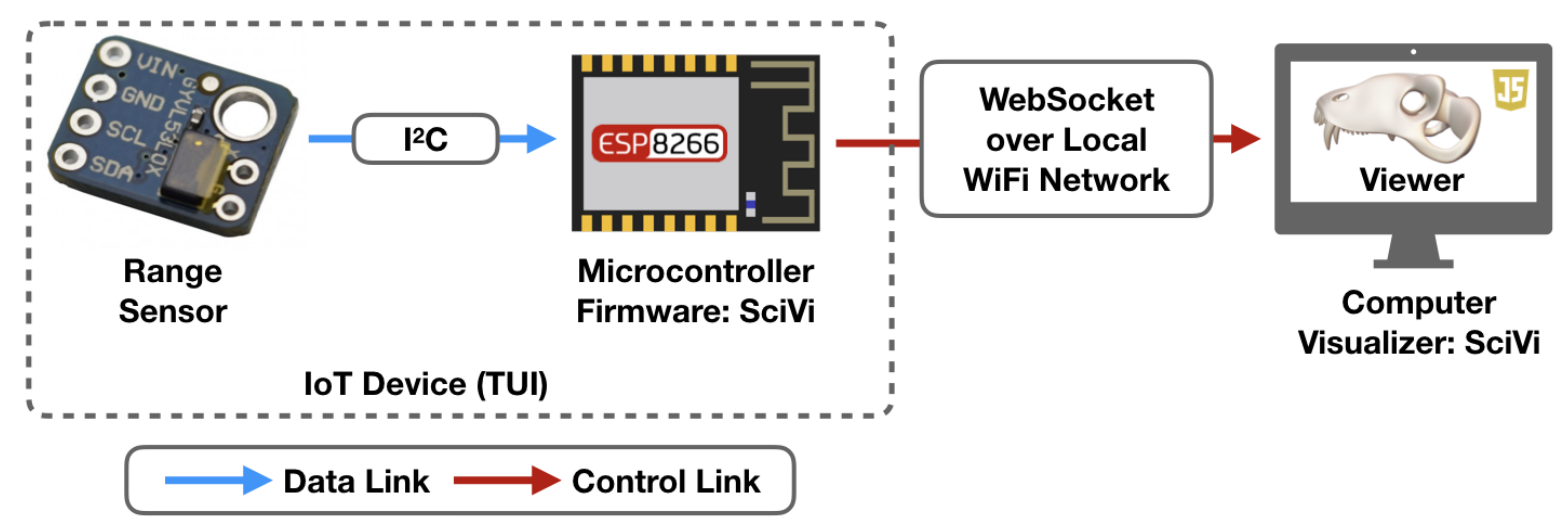

Fig. 10. Operating schema of the "Titanophone" cyber-physical exhibit.

SciVi system was used to generate both the firmware for the ESP8266 microcontroller and the visualization client. The firmware was generated in $\mathrm{C}++$ and contains the following main steps:

1. Start up the WiFi access point (SSID and password are set up in SciVi when tuning the firmware generation).

2. Start main loop:

a. Poll the range sensor (either VL53LoX connected to ESP8266 via $\mathrm{I}^{2} \mathrm{C}$, or HC-SRo4 connected to digital input pins).

b. Transmit the distance as JSON message via WebSocket connection over WiFi to the visualization client.

The visualization client was generated in JavaScript, HTML5 and CSS as a standalone Web page. The rendering is based on Three.js engine [27]. The following main actions are performed on the client:

1. By loading:

a. Load the models of No. 157/1 and No. 157/3 skulls (stored as static resources in Stanford triangle format [28]). 
b. Initialize morph targets [29] from the loaded models. As mentioned above, the topologies of both models are equal, so the simple shape morphing is possible. This kind of morphing is supported out of the box by Three.js engine.

c. Connect to the IoT device via local WiFi utilizing standard WebSocket API of the browser. The IP address is static, because the IoT device is a hotspot. 2. By receiving the message via WebSocket from the IoT device:

a. If using HC-SRo4 range sensor, perform moving average smoothing for the measurements to compensate jitter. If using VL53LoX range sensor, take the measurements as they are, because this sensor compensates jitter itself.

b. Calculate the morphing parameter $t$. It is assumed, that the skulls are at $40 \mathrm{~cm}$ distance from each other, so to get $t$ the measured range is divided by 40 and clamped to $[0 ; 1]$.

c. Render the morphed model based on loaded morph targets and calculated $t$ parameter.

All the needed software components, like the support of range sensor and morph targets were integrated into SciVi by extending related ontologies (the ontology of electronic components and the ontology of visual objects). The demo of the created exhibit is accessible online [30] and shown in the Fig. 11. In this demo, the physical part is replaced by the slider on the bottom of the page. Moving this slider, the user can choose the intermediate ontogenetic stages of titanophone.
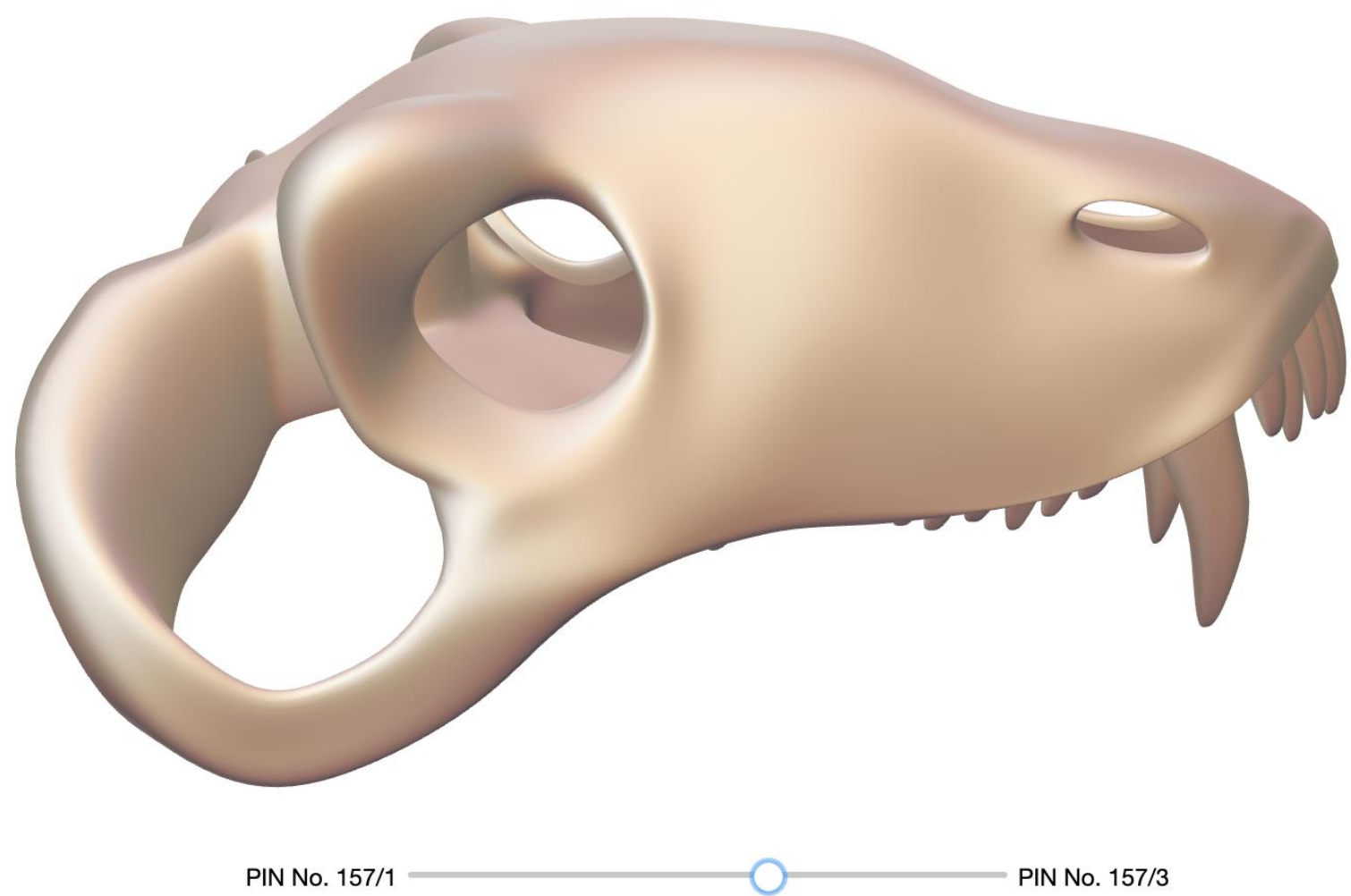

Fig. 11. "Titanophone" cyber-physical exhibit online demo. 


\section{Conclusion}

As a result of the reported research, the concept of deploying CPS in the museum space was proposed. The key idea of this concept is the use of the adaptive multiplatform scientific visualization and visual analytics system SciVi. The adaptive mechanisms of this system allow using it as an efficient middleware for enriching the museum's digital infrastructure with the new multimedia content management tools. Thus, seamless integration of new interactive features into the existing museum exhibition is achieved, for example, the creation of tangible interfaces to existing museum objects based on IoT technologies. The scientific visualization methods of SciVi ensure scientifically correct rendering of the data contributing to the reliability of presenting the multimedia content related to the exhibit.

The viability and efficiency of the proposed concept has been tested in practice by creating two cyber-physical museum exhibits: bonobo monkey skull in the State Darwin Museum and titanophone skulls in the Museum of Permian Antiquities. The use of TUI in these exhibits allowed to expand the range of information they provide by supporting haptic interaction. This increases the attractiveness of corresponding museum objects and contributes to the museum inclusion, making these objects accessible by visually impaired visitors.

In the future we plan to study the issues of protecting wireless cyber-physical exhibits from theft, as well as further enrichment of the digital infrastructure of museums with tangible user interfaces.

\section{Acknowledgements}

We thank Aurore Mathys from the Royal Museum of Central Africa (Tervuren, Belgium), who kindly provided a 3D model of the bonobo skull.

We thank Constantine Tarasenko, Andrey Sennikov, Valeriy Golubev from the Paleontological Institute, Russian Academy of Sciences (Moscow, Russia), who kindly provided the access to the titanophone holotype and lectotype.

\section{References}

1. Ishii, H., Ullmer, B. Tangible Bits: Towards Seamless Interfaces Between People, Bits and Atoms // CHI '97 Proceedings of the ACM SIGCHI Conference on Human Factors in Computing Systems. - ACM, 1997. - PP. 234-241. DOI: 10.1145/258549.258715.

2. Rose, K., Eldridge, S., Chapin, L. The Internet of Things: an Overview [Electronic Resource] // The Internet Society (ISOC). - 2015. URL: https://www.internetsociety.org/resources/doc/2015/iot-overview (last accessed 14.10.2019).

3. Sanfelice, R. Analysis and Design of Cyber-Physical Systems. A Hybrid Control Systems Approach // Cyber-Physical Systems: From Theory to Practice / Rawat D., Rodrigues J., Stojmenovic I. - CRC Press, 2015. - PP. 3-31. DOI: 10.1201/b19290-3.

4. Marr, B. Why Everyone Must Get Ready For The 4th Industrial Revolution [Electronic Resource] // Forbes. - $2016 . \quad$ URL: https://www.forbes.com/sites/bernardmarr/2016/04/05/why-everyone-mustget-ready-for-4th-industrial-revolution/ (last accessed 14.10.2019).

5. Chianese, A., Piccialli, F. Designing a Smart Museum: when Cultural Heritage Joins IoT // Third International Conference on Technologies and Applications for Smart Cities (I-TASC'14). - 2014. - 7 p. DOI: 10.1109/NGMAST.2014.21. 
6. Ryabinin, K., Chuprina S., Belousov K. Ontology-Driven Automation of IoT-Based Human-Machine Interfaces Development // Lecture Notes in Computer Science. - Springer, 2019. - Vol. 11540. - P. 110-124. DOI: 10.1007/978-3-030-22750o_9.

7. Ryabinin, K., Kolesnik, M. Using IoT Devices Powered by Scientific Visualization Tools to Create Interactive Paleontological Museum Exhibitions // Proceedings of $28^{\text {th }}$ International Conference on Computer Graphics and Vision "GraphiCon 2018”. - Tomsk, 2018. - PP. 70-73.

8. Hirose K. Research on Methods of "Touching the World" - The Aim of the Exhibit Area of Tactile Learning in Japan's National Museum of Ethnology // Disability Studies Quarterly. - 2013. - Vol. 33, No. 3. DOI: 10.18061/dsq.v33i3.3743.

9. Alboul, L., Beer, M., Nisiotis, L. Merging Realities in Space and Time: Towards a New Cyber-Physical Eco-Society // Cyber-Physical Systems for Social Applications / Dimitrova, M., Wagatsuma, H. - IGI Global, 2019. - PP. 156-183.

10. Fischer, T., Herr, C.M., Burry, M.C., Frazer, J.H. Tangible Interfaces to Explain Gaudi's Use of Ruled-Surface Geometries Interactive Systems Design for Haptic, Nonverbal Learning // Automation in Construction. - Elsevier, 2003. - PP. 467471. DOI: 10.1016/So926-5805(03)oo031-1.

11. Hsieh, C., Liu, I., Yu, N., Chiang, Y., Wu, H., Chen, Y., Hung, Y. Yongzheng Emperor's Interactive Tabletop: Seamless Multimedia System in a Museum Context // Proceedings of the International Conference on Multimedia. - ACM, 2010. PP. 1453-1456. DOI: 10.1145/1873951.1874242.

12. Muntean, R., Hennessy, K., Antle, A., Rowley, S., Wilson, J., Matkin, B., Eckersley, R., Tan, P., Wakkary, R. Belongings: a Tangible Interface for Intangible Cultural Heritage // Proceedings of the Conference on Electronic Visualisation and the Arts. - British Computer Society, 2015. - PP. 360-366. DOI: 10.14236/ewic/eva2015.41.

13. Okerlund, J., Segreto, E., Grote, C., Westendorf, L., Scholze, A., Littrell, R., Shaer, O. SynFlo: A Tangible Museum Exhibit for Exploring Bio-Design // Proceedings of the TEI'16: Tenth International Conference on Tangible, Embedded, and Embodied Interaction 2016. - ACM, 2016. - PP. 141-149. DOI: 10.1145/2839462.2839488

14. Vaz, R., Fernandes, P., Veiga A. Proposal of a Tangible User Interface to Enhance Accessibility in Geological Exhibitions and the Experience of Museum Visitors // Procedia Computer Science. - Elsevier, 2016. - Vol. 100. - PP. 832-839. DOI: 10.1016/j.procs.2016.09.232..

15. Vaz, R., Fernandes, P., Veiga A. Designing an Interactive Exhibitor for Assisting Blind and Visually Impaired Visitors in Tactile Exploration of Original Museum Pieces // Procedia Computer Science. - Elsevier, 2018. - Vol. 138. - PP. 561-570. DOI: 10.1016/j.procs.2018.10.076.

16. Hassanin, H., Jiang, K. Chapter 10 - Net Shape Manufacture of Freestanding Ceramic Micro-components through Soft Lithography // Micromanufacturing Engineering and Technology (Second Edition). - Elsevier, 2015. - PP. 239-256. DOI:10.1016/B978-0-323-31149-6.00010-4.

17. Ryabinin, K., Chuprina, S. High-Level Toolset for Comprehensive Visual Data Analysis and Model Validation // Procedia Computer Science. - Elsevier, 2017. Vol. 108. - PP. 2090-2099. DOI: 10.1016/j.procs.2017.05.050.

18. Bonobo Photo Exhibition [Electronic Resource] // State Darwin Museum. 2018. URL: http://www.darwinmuseum.ru/projects/exhibition/bonobo (last accessed 14.10.2019). 
19. Royal Museum for Central Africa [Electronic Resource]. URL: https://www.africamuseum.be/ (last accessed 14.10.2019).

20.Pan paniscus Skull by Royal Museum for Central Africa [Electronic Resource] // Sketchfab. URL: https://sketchfab.com/3d-models/pan-paniscus-skullb7ba07ddcooc4572bcd7a6aof6faecec (last accessed 14.10.2019).

21. Mahony, R., Hamel, T., Pflimlin J. Nonlinear Complementary Filters on the Special Orthogonal Group // IEEE Transactions on Automatic Control. - IEEE, 2008. - Vol. 53, No. 5. - PP. 1203-1218. DOI: 10.1109/TAC.2008.923738.

22. Sketchfab Viewer API [Electronic Resource] // Sketchfab. URL: https://sketchfab.com/developers/viewer (last accessed 14.10.2019).

23. Akhtamzyan, A. Ryabinin, K., Olshansky, D., Sudarikova, E. Creating an Interactive Museum Exhibit using an IoT Joystick [Electronic Resource] // Sketchfab. 2019. URL: https://sketchfab.com/blogs/community/creating-an-interactivemuseum-exhibit-using-an-iot-joystick/ (last accessed 14.10.2019).

24. Efremov, I. Some New Permian Reptiles of the USSR // Compt. Rend. (Dok) Acad. Sci. USSR. Paleontol. - 1938. - Vol. 19, No. 9. - P. 771-776.

25. Ivakhnenko, M. Eotherapsids from the East European Placket // Paleontological Journal. - 2003. - No. 37. - PP. 339-465.

26. Kolesnik, M. Modern Reconstruction Of Titanophoneus Potens Efremov (Synapsida. Dinocephalia) // The University of Opole. MSc Thesis. - Opole, 2019. - 60 p. URL: http://apd.uni.opole.pl/ (last accessed 14.10.2019).

27. Three.js Graphics Rendering Engine [Electronic Resource]. URL: https://threejs.org/ (last acceessed 13.10.2019).

28. Bourke, P. PLY - Polygon File Format [Electronic Resource]. URL: http://paulbourke.net/dataformats/ply/ (last accessed 14.10.2019).

29. Geometry Morph Targets [Electronic Resource] // Three.js Graphics Rendering Engine. URL: https://threejs.org/docs/\#api/en/core/Geometry.morphTargets (last accessed 14.10.2019).

30. Ryabinin, K., Kolesnik, M. Titanophone Cyber-Physical Exhibit Demo // SciVi Platform. - 2019. URL: https://scivi.tools/titanophone/ (last accessed 14.10.2019). 